\title{
Introduction to the Theory of Critical Phenomena
}


This page is intentionally left blank 


\section{INTRODUCTION TO THE}

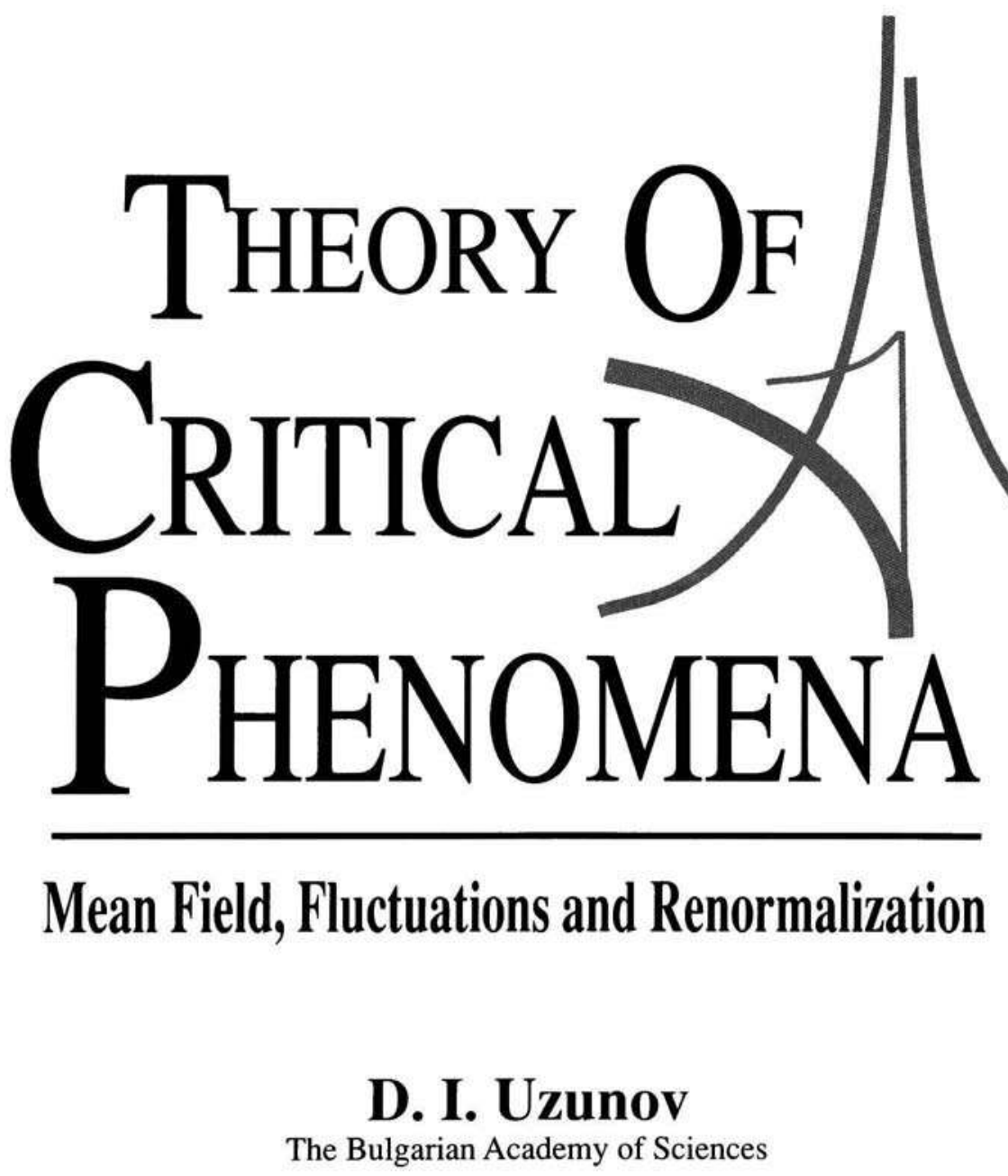

The Bulgarian Academy of Sciences 


\title{
Published by
}

World Scientific Publishing Co. Pte. Ltd.

P O Box 128, Farrer Road, Singapore 9128

USA office: Suite 1B, 1060 Main Street, River Edge, NJ 07661

UK office: 73 Lynton Mead, Totteridge, London N20 8DH

\section{INTRODUCTION TO THE THEORY OF CRITICAL PHENOMENA}

Copyright (c) 1993 by World Scientific Publishing Co. Pte. Ltd.

All rights reserved. This book, or parts thereof, may not be reproduced in any form or by any means, electronic or mechanical, including photocopying, recording or any information storage and retrieval system now known or to be invented, without written permission from the Publisher.

\author{
ISBN 981-02-0388-8 \\ 981-02-0389-6 (pbk)
}

Printed in Singapore by JBW Printers \& Binders Pte. Ltd. 
To DIANA

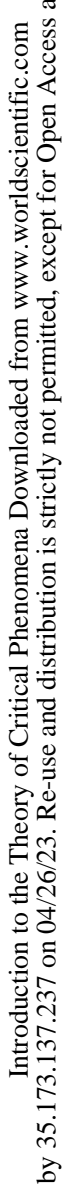


This page is intentionally left blank 


\section{Foreword}

It is a pleasure for me to present to the public this monograph on phase transitions and critical phenomena. In the past few years, along with our improving understanding of these phenomena, their theoretical description has made great progress evolving into more advanced forms. These also include, apart from new approximations elaborated in the framework of the mean-field theory, various novel approaches such as phenomenological and microscopic treatments of fluctuations, the scaling theory and the renormalization group theory. All of them are thoroughly explained in the present book. The author has managed to cover all these topics with a good balance, proceeding from the thermodynamics of phase transitions to the modern theory of the renormalization group. The way of presentation adopted by the author is very instructive. Detailed derivations of almost all the basic equations are included in the monograph. Thus, even readers who are too lazy or too busy to reproduce such calculations themselves can follow easily the discourse to its very end.

Even the introduction of the renormalization group theory does not diminish the importance of the mean-field theory, as we can see, for example, from the recent development of the coherent-anomaly method. Hence, the meticulous explanation of this theory in the present book seems to be justified even from the viewpoint of modern approaches to critical phenomena. Chapter 6 on fluctuations and Chapter 7 on the perturbation theory of fluctuation fields constitute a good introduction to Chapter 8 on the renormalization group theory. The last chapter contains a brief overview of some important applications of the latter method to various problems including the Bose gas, the classical-to-quantum crossover, the random-field problem, the anisotropy effect and multicritical behavior. This part of the book 
displays considerable expertise as Dr. Uzunov himself has made a series of interesting contributions in this field.

\author{
Masuo Suzuki \\ Professor of Statistical Physics \\ University of Tokyo
}




\section{Preface}

This book is intended as an introduction to the theory of phase transitions and critical phenomena. The phase transitions are described by the methods of thermodynamics and statistical physics. On the contrary modern methods for the investigation of outstanding problems in statistical physics, thermodynamics, condensed matter physics and field theory may be partly ascribed to the development of the ideas and theoretical techniques intended to solve the questions of phase transitions. The sophistication of theoretical methods is often an obstacle to the quick understanding of important results currently reported in scientific literature. The main purpose of this book is to present a part of these methods in an intelligible form for students.

The physics of phase transitions is old and abundant in fascinating ideas, theories and experiments. Most of them are substantial for other fields in physics, chemical physics and even for other natural sciences. All the problems concerning phase transitions and even the main part of them cannot be comprised in only one book. I have chosen such a representation of the phase transition theory that goes along a general scheme for the description of phase transitions and critical phenomena. It includes the following topics: the thermodynamic stability theory (Chapter 2) and its connection with the classical and scaling picture of the phase transitions (Chapter 3); the Landau expansion as a further development of the thermodynamic approach and the notion of order and symmetry in condensed matter physics (Chapter 4); an introduction to statistical mechanics and the mean field approximation for homogeneous and inhomogeneous systems (Chapter 5); the fluctuation phenomena and the renormalization group method in treating the fluctuation fields (Chapters 6-8). I have made an attempt to establish a logical relationship between the different theories and their role in the interpretation of experiments. Special attention is paid to the problems which are less illuminated in books and review articles published in the last twenty years. On the contrary, I have only mentioned some important problems like the phenomenological scaling 
and the dynamic critical phenomena, for which several excellent books and reviews exist. The text contains a number of references to the literature intended for the reader who is interested in specific problems.

In addition to the presentation of basic ideas, a big part of Chapters 2-8 is devoted to the concrete methods of calculation, characterizing the different theories and approximations. This especially concerns Chapter 7 , where the perturbation theory of fluctuations is discussed in detail. I suppose that in this way the renormalization group approach will be more easily comprehended both as a concept and as a technique of calculation.

In Chapters 2-6, I often follow the deductive way of presentation, which to my opinion reveals the wide application of the quasiphenomenological theory to the description of phase transitions.

Chapters 7 and 8 are written in an opposite manner, since when the perturbation theory and diagrammatic technique are understood for simple models, their generalization to more complex cases can be made without difficulties.

Chapters 1 and 9 have in some sense a different role. In Chapter 1 the main stages in the development of theoretical and experimental studies on the phase transitions are outlined accompanied by corresponding references to the literature. The unexperienced reader may find this difficult. In this case I recommend reading it after an acquaintance with the contents of the remaining chapters. Chapter 9 presents the application of the mean field and fluctuation theories to concrete problems. In order to keep this survey within a manageable length it was necessary to avoid, as much as possible, problems which are widely discussed in other reviews. My intention has been to throw light on issues, which are not reviewed in the existing literature. Even within this limited scope it was impossible to present within a single chapter a detailed discussion of all developments; many interesting contributions and details of the technique of calculation are given in the numerous references to original papers. The predominant part of Chapter 9 can be thought as a sum of exercises together with a brief introduction to the problems and a short discussion of the main results. I hope that this part of the book may be interesting also to specialists working in the field of phase transitions.

In the Appendix, I give some mathematical details about the methods for calculation of the functional expansions and perturbation integrals.

This book would not have been completed without the substantial support of Prof. G. Scarpetta and the colleagues from the Department of Theoretical Physics at Salerno University and G. Nadjakov Institute of Solid State Physics of the Bulgarian Academy of Sciences in Sofia. I want to thank my colleagues, the stimulating discussions with them have improved my understanding of the problems of the phase transition theory. They are: Y. Brankov, M. Bushev, K. Chao, L. De Cesare, M. E. Fisher, R. Folk, V. L. Ginzburg, B. I. Halperin, J. A. Hertz, H. Iro, I. D. Lawrie, R. Micnas, L. P. Pitaevskii, V. L. Pokrovskii, D. I. Pushkarov, Kh. Pushkarov, I. Sznajd, N. S. Tonchev, M. Suzuki, and M. Zannetti.

The critical reading of parts of the manuscript by Ms. E. J. Blagoeva, Prof. L. De Cesare, Dr. Y. T. Millev, Dr. C. Noce, Dr. I. Rabuffo, and Dr. A. Romano as well as the technical assistance by Drs. A. Saggese and V. Scarano in the final 
preparation of the camera-ready version of the book are gratefully acknowledged.

I am grateful for the hospitality of the Salerno University where this book has been written.

Salerno, January 1992

D. I. Uzunov 
This page is intentionally left blank 


\section{Contents}

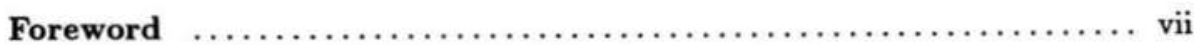

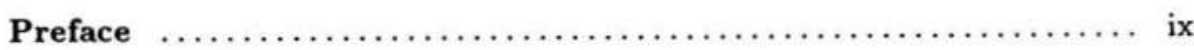

Chapter 1 Introduction $\ldots \ldots \ldots \ldots \ldots \ldots \ldots \ldots \ldots \ldots \ldots \ldots \ldots \ldots, 1$

Chapter 2 Equilibrium States and Phases $\ldots \ldots \ldots \ldots \ldots \ldots \ldots \ldots \ldots \ldots$

$2.1 \quad$ Phases $\ldots \ldots \ldots \ldots \ldots \ldots \ldots \ldots \ldots \ldots \ldots \ldots \ldots \ldots, 18$

$2.2 \quad$ Fundamental relations $\ldots \ldots \ldots \ldots \ldots \ldots \ldots \ldots \ldots \ldots \ldots \ldots, 19$

2.2.1 Fundamental equations $\ldots \ldots \ldots \ldots \ldots \ldots \ldots, 20$

2.2.2 Properties $\ldots \ldots \ldots \ldots \ldots \ldots \ldots \ldots \ldots \ldots \ldots, 20$

2.2.3 Notations $\ldots \ldots \ldots \ldots \ldots \ldots \ldots \ldots \ldots \ldots \ldots, 22$

2.2.4 Macroscopic scales $\ldots \ldots \ldots \ldots \ldots \ldots \ldots \ldots, 23$

$2.3 \quad$ Entropy increase $\ldots \ldots \ldots \ldots \ldots \ldots \ldots \ldots \ldots \ldots \ldots \ldots \ldots \ldots$

2.4 Conditions of equilibrium and stability $\ldots \ldots \ldots \ldots \ldots 26$

2.4.1 Equilibrium conditions $\ldots \ldots \ldots \ldots \ldots \ldots \ldots 27$

2.4 .2 Stability conditions $\ldots \ldots \ldots \ldots \ldots \ldots \ldots \ldots \ldots \ldots \ldots$

$2.5 \quad$ Classification of equilibria $\ldots \ldots \ldots \ldots \ldots \ldots \ldots \ldots \ldots \ldots, 34$

2.6 Thermodynamic potentials $\ldots \ldots \ldots \ldots \ldots \ldots \ldots \ldots \ldots \ldots \ldots \ldots$

2.6.1 Energy minimum $\ldots \ldots \ldots \ldots \ldots \ldots \ldots \ldots, 36$

2.6.2 Legendre transformations and extremum conditions $\ldots \ldots \ldots \ldots \ldots \ldots \ldots \ldots \ldots \ldots, 38$

2.6.3 Legendre transformations in entropy representation $\ldots \ldots \ldots \ldots \ldots \ldots \ldots \ldots \ldots \ldots, 40$

2.6.4 Application $\ldots \ldots \ldots \ldots \ldots \ldots \ldots \ldots \ldots, 41$

$2.7 \quad$ Coexistence of phases $\ldots \ldots \ldots \ldots \ldots \ldots \ldots \ldots \ldots \ldots, 43$

2.7.1 Equilibrium conditions $\ldots \ldots \ldots \ldots \ldots \ldots \ldots, 44$

2.7.2 Gibbs phase rule $\ldots \ldots \ldots \ldots \ldots \ldots \ldots \ldots, 45$ 
2.7.3 Stability conditions

2.7.4 Limitation of the thermodynamic theory of stability

Chapter 3 Thermodynamic Theory of Phase Transitions $\ldots \ldots \ldots 51$

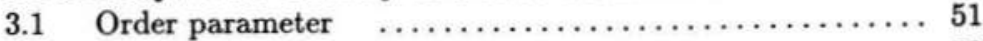

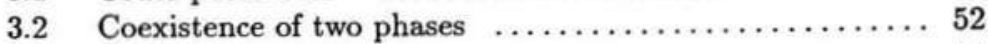

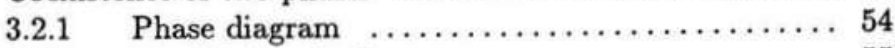

3.2.2 Equilibrium isotherms $\ldots \ldots \ldots \ldots \ldots \ldots \ldots \ldots, 55$

3.2.3 Relations on the coexistence line $\ldots \ldots \ldots \ldots .56$

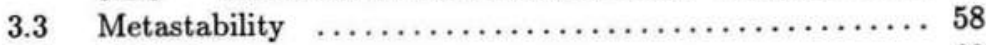

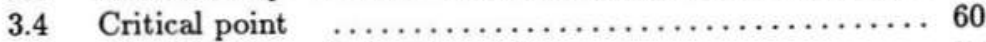

3.5 Classifications of phase transitions $\ldots \ldots \ldots \ldots \ldots \ldots \ldots 63$

3.6 Compressible systems $\ldots \ldots \ldots \ldots \ldots \ldots \ldots \ldots \ldots \ldots \ldots 6 . \ldots \ldots$

3.6.1 Gas-liquid-solid diagram $\ldots \ldots \ldots \ldots \ldots \ldots \ldots 66$

3.6.2 The solid phase $\ldots \ldots \ldots \ldots \ldots \ldots \ldots \ldots \ldots \ldots \ldots$

3.6.3 The liquid state $\ldots \ldots \ldots \ldots \ldots \ldots \ldots \ldots \ldots \ldots \ldots \ldots \ldots$

3.7 Phase separation in mixtures $\ldots \ldots \ldots \ldots \ldots \ldots \ldots \ldots \ldots \ldots \ldots \ldots$

3.7.1 Binary mixtures $\ldots \ldots \ldots \ldots \ldots \ldots \ldots \ldots \ldots \ldots \ldots \ldots \ldots \ldots .74$

3.7.2 Stability conditions and critical states in binary mixtures $\ldots \ldots \ldots \ldots \ldots \ldots \ldots \ldots \ldots \ldots \ldots \ldots$

3.7.3 Coexistence surfaces, critical lines, and second Gibbs rule $\ldots \ldots \ldots \ldots \ldots \ldots \ldots \ldots 77$

3.7.4 Tricritical and critical end points.

Multicritical phenomena ................ 78

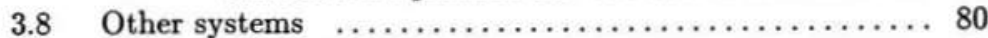

3.8.1 General notations $\ldots \ldots \ldots \ldots \ldots \ldots \ldots \ldots .60$

3.8.2 Ferromagnets ..................... 82

3.8.3 Anisotropy in ferromagnets $\ldots \ldots \ldots \ldots \ldots \ldots .66$

3.8.4 Complex magnetic order ............... 86

3.8.5 Structural and ferroelectric phase transitions ... 90

3.8.6 Superfluidity and superconductivity ........ 92

3.8.7 Liquid crystals $\ldots \ldots \ldots \ldots \ldots \ldots \ldots \ldots \ldots . . \ldots \ldots$

3.9 Advantages and disadvantages of the thermodynamic

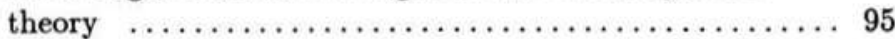

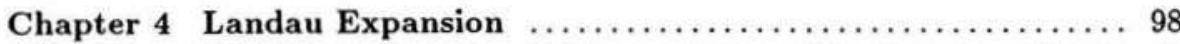

$4.1 \quad$ Introductory remarks $\ldots \ldots \ldots \ldots \ldots \ldots \ldots \ldots \ldots \ldots \ldots$

$4.2 \quad$ Appropriate variables $\ldots \ldots \ldots \ldots \ldots \ldots \ldots \ldots \ldots \ldots \ldots$

4.3 Generalized Gibbs potential $\ldots \ldots \ldots \ldots \ldots \ldots \ldots \ldots \ldots \ldots$

4.3.1 Nonequilibrium potential $F^{t} \ldots \ldots \ldots \ldots \ldots \ldots .101$

4.3.2 Nonequilibrium potential $\Phi$............. 102

4.3.3 Large reservoir and relation between

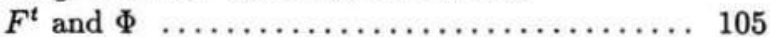

4.4 Landau potential $\ldots \ldots \ldots \ldots \ldots \ldots \ldots \ldots \ldots \ldots \ldots \ldots \ldots \ldots$ 
4.4.1 Taylor expansion $\ldots \ldots \ldots \ldots \ldots \ldots \ldots \ldots \ldots$

4.4.2 Equilibrium $\ldots \ldots \ldots \ldots \ldots \ldots \ldots \ldots \ldots \ldots \ldots \ldots$

4.4.3 Stability .......................... 107

4.4.4 Aims of the Landau theory $\ldots \ldots \ldots \ldots \ldots \ldots$

4.4.5 Examples ........................ 109

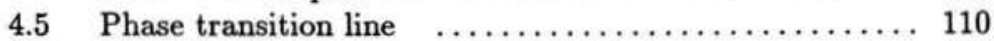

4.6 Second order phase transitions $\ldots \ldots \ldots \ldots \ldots \ldots \ldots \ldots \ldots \ldots$

4.7 First order transitions and the isolated critical point $\ldots 116$

4.7.1 Phase diagram of the first order transition .... 116

4.7.2 Metastability. Specific heat $\ldots \ldots \ldots \ldots \ldots \ldots \ldots \ldots$

4.7.3 The isolated critical point $\ldots \ldots \ldots \ldots \ldots \ldots .120$

4.8 Extended expansion: $\varphi^{6}$ - and $\varphi^{8}$-theories $\ldots \ldots \ldots \ldots \ldots 121$

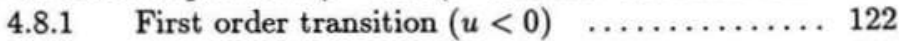

4.8.2 The tricritical point $(u=0) \ldots \ldots \ldots \ldots \ldots \ldots \ldots \ldots \ldots$

4.8.3 Irrelevance of the $\varphi^{6}$-term for $u>0 \ldots \ldots \ldots 127$

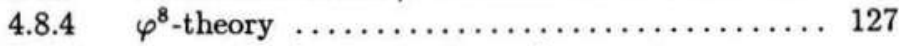

4.9 Multicomponent order parameter and symmetry

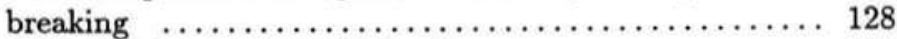

4.9.1 Thermodynamic functions $\ldots \ldots \ldots \ldots \ldots \ldots \ldots$

4.9.2 Symmetry breaking and symmetry conserving phase transitions. Landau criteria for second order phase transitions $\ldots \ldots \ldots \ldots 129$

4.10 Effect of external field and susceptibility $\ldots \ldots \ldots \ldots \ldots 131$

4.10.1 One-component order parameter .......... 131

4.10.2 Two-component order parameter $\ldots \ldots \ldots \ldots .135$

4.11 Miscellaneous topics $\ldots \ldots \ldots \ldots \ldots \ldots \ldots \ldots \ldots \ldots \ldots \ldots \ldots$

4.11.1 $\begin{aligned} & \text { Notion of crossover phenomena. } \\ & \text { Critical-to-tricritical crossover } \ldots \ldots \ldots \ldots \ldots 137\end{aligned}$

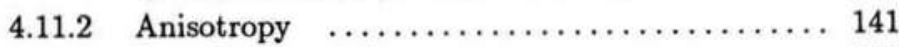

4.11.3 Coupling to strains $\ldots \ldots \ldots \ldots \ldots \ldots \ldots \ldots \ldots \ldots \ldots \ldots \ldots$

4.11.4 Validity and further development of the

Landau theory $\ldots \ldots \ldots \ldots \ldots \ldots \ldots \ldots \ldots \ldots \ldots$

Chapter 5 Statistical Mechanics and Mean-Field

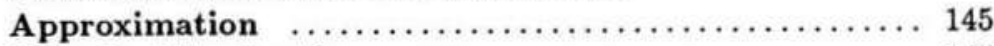

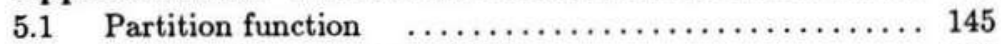

5.1.1 Microscopic variables $\ldots \ldots \ldots \ldots \ldots \ldots \ldots . \ldots \ldots$

5.1.2 Canonical distribution $\ldots \ldots \ldots \ldots \ldots \ldots \ldots, 146$

5.1.3 Thermodynamic relations ............... 148

5.2 Fixed external field and order parameter fluctuations .. 149

5.2.1 Continuous variable $\varphi \ldots \ldots \ldots \ldots \ldots \ldots \ldots \ldots$

5.2.2 Discrete variable $\varphi \quad \ldots \ldots \ldots \ldots \ldots \ldots \ldots \ldots \ldots \ldots \ldots \ldots \ldots$

5.3 Inhomogeneous systems and translational invariance $\ldots 153$

5.4 Coarse-graining and effective Hamiltonian $\ldots \ldots \ldots \ldots .154$

$5.4 .1 \quad$ Coarse-graining $\ldots \ldots \ldots \ldots \ldots \ldots \ldots \ldots \ldots \ldots \ldots \ldots$ 
5.4.2 Transformation of the canonical partition

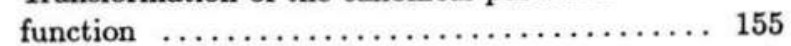

5.4.3 Quasimacroscopic separability ........... 157

5.4.4 Separability of the Hamiltonian $\ldots \ldots \ldots \ldots . . \ldots 159$

5.4.5 Effective Hamiltonian $\ldots \ldots \ldots \ldots \ldots \ldots \ldots$

5.4.6 Remarks ....................... 161

5.5 Generating functionals and correlation functions $\ldots \ldots \ldots 162$

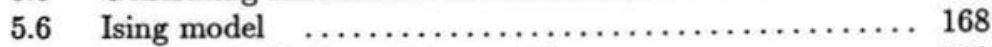

5.6.1 Definition of the model ................. 168

5.6.2 Generalization and remarks $\ldots \ldots \ldots \ldots \ldots \ldots . \ldots 169$

5.7 Other models $\ldots \ldots \ldots \ldots \ldots \ldots \ldots \ldots \ldots \ldots \ldots \ldots \ldots \ldots \ldots \ldots \ldots$

5.7.1 Heisenberg and $X Y$ model $\ldots \ldots \ldots \ldots \ldots \ldots \ldots \ldots \ldots \ldots$

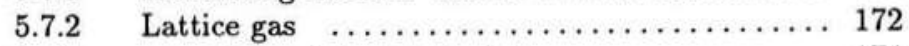

5.7.3 n-component vector models ............ 174

5.7.4 The $s$-state Potts model .................. 174

5.7.5 The spherical model .................. 174

$\begin{array}{llll}\text { 5.7.6 Biquadratic exchange and single spin energy } & \text {.. } & 175\end{array}$

5.8 Mean-field approximation $\ldots \ldots \ldots \ldots \ldots \ldots \ldots \ldots \ldots \ldots \ldots \ldots \ldots$

$5.8 .1 \quad$ Mean field (MF) $\ldots \ldots \ldots \ldots \ldots \ldots \ldots \ldots \ldots \ldots \ldots$

5.8.2 Thermodynamic potential $\ldots \ldots \ldots \ldots \ldots \ldots . .177$

$\begin{array}{llll}5.8 .3 & \text { Uniform systems and equation of state } \ldots \ldots & 179\end{array}$

5.8.4 Expansion of the equation of state and the critical point $\ldots \ldots \ldots \ldots \ldots \ldots \ldots \ldots \ldots \ldots \ldots \ldots \ldots \ldots$

5.8.5 Helmholtz free energy and generalized potential .......................... 182

5.9 Condensation and statistical mechanics $\ldots \ldots \ldots \ldots \ldots .183$

5.9.1 On the derivation of the van der Waals equation $\ldots \ldots \ldots \ldots \ldots \ldots \ldots \ldots \ldots \ldots \ldots \ldots \ldots \ldots$

5.9.2 Corresponding states and universality $\ldots \ldots \ldots 185$

5.9.3 Critical properties of van der Waals fluids .... 186

Chapter 6 Fluctuations and Fields $\ldots \ldots \ldots \ldots \ldots \ldots \ldots \ldots \ldots \ldots \ldots$

6.1 Description of macroscopic fluctuations .......... 188

6.2 Spatially independent fluctuations and anisotropy $\ldots \ldots \quad 190$

6.3 Partition function of spatially independent

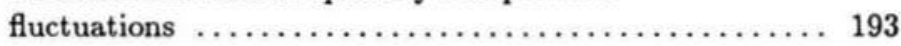

6.3.1 Gaussian fluctuations $\ldots \ldots \ldots \ldots \ldots \ldots \ldots . \ldots 194$

6.3.2 Beyond the Gaussian approximation. Weak coupling .................... 196

6.3.3 Strong coupling and closed-form expression of the partition integral $\ldots \ldots \ldots \ldots \ldots \ldots 200$

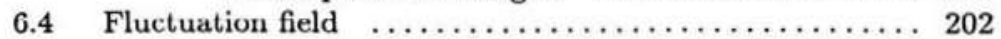

6.5 From lattice to field models and vice versa ......... 207

6.5.1 Continuum limit $\ldots \ldots \ldots \ldots \ldots \ldots \ldots \ldots \ldots \ldots \ldots \ldots$ 
6.5.2 The origin of fields in the generalized thermodynamics ...................... 209

6.5.3 Discretization of field models ............ 210

6.5.4 Continuum limit in the momentum space ..... 211

6.5.5 Functional integral $\ldots \ldots \ldots \ldots \ldots \ldots \ldots \ldots .213$

6.5.6 Abbreviated series ................... 214

6.6 Integral transformation of microscopic models ........ 215

6.6.1 Transformation of the Ising model and relation to the mean field theory $\ldots \ldots \ldots \ldots \ldots 216$

6.6.2 Transformation of other models ........... 223

6.7 Short-range and long-range translationally invariant

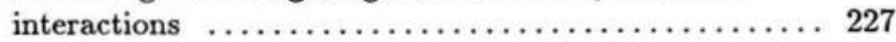

Chapter 7 Perturbation Theory of Fluctuation Fields . . . . . . 231

7.1 Basic fluctuation Hamiltonian $\ldots \ldots \ldots \ldots \ldots \ldots \ldots \ldots \ldots \ldots$

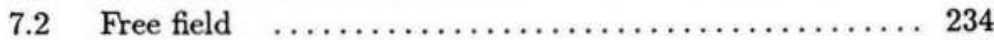

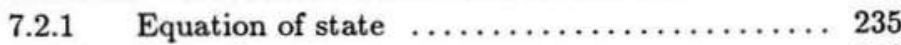

7.2.2 Green function $\ldots \ldots \ldots \ldots \ldots \ldots \ldots \ldots \ldots \ldots \ldots \ldots \ldots \ldots \ldots \ldots$

7.3 Thermodynamic properties of free fields $\ldots \ldots \ldots \ldots \ldots 240$

7.4 Correlation function of the free fields $\ldots \ldots \ldots \ldots \ldots .244$

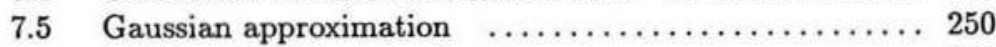

7.5.1 Goldstone modes ................... 251

7.5.2 Ginzburg-Levanyuk criterion and critical

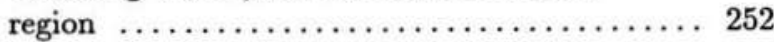

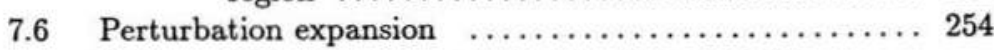

7.6.1 Perturbation expansion for the partition function and the thermodynamic potential .... 255

7.6.2 Perturbation expansion for the averages ...... 260

$7.7 \quad \varphi^{4}$-theory. Coordinate representation $\ldots \ldots \ldots \ldots \ldots \ldots 261$

7.7.1 First order perturbation contribution to the thermodynamic potential $\ldots \ldots \ldots \ldots \ldots \ldots, 262$

7.7.2 Perturbation contribution to the correlation

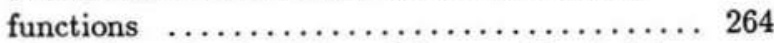

7.7.3 Remarks and calculation of the combinatorial factors ........................... 269

$7.8 \quad \varphi^{4}$-theory. Momentum representation $\ldots \ldots \ldots \ldots \ldots \ldots 272$

7.8.1 First-order perturbation terms $\ldots \ldots \ldots \ldots \ldots 273$

7.8.2 Second-order perturbation terms ......... 275

7.9 Dyson equation and self-energy function $\ldots \ldots \ldots \ldots \ldots 278$

7.9.1 Dyson equation .................... 279

7.9.2 Expansion of the self-energy function to

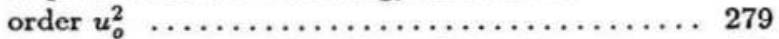

7.9.3 Remarks on the reducibility of the diagrams .. 282

7.10 Notion of renormalization $\ldots \ldots \ldots \ldots \ldots \ldots \ldots \ldots \ldots \ldots \ldots \ldots$ 
7.10.1 Mass renormalization from the perturbation

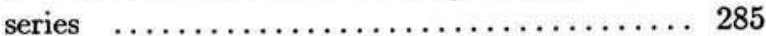

7.10.2 Mass-renormalization counter-term ........ 286

7.11 Properties of the standard perturbation series ........ 289

7.11.1 How to determine the critical region from the perturbation expansion $\ldots \ldots \ldots \ldots \ldots \ldots 289$

7.11.2 Effective expansion parameter ........... 292

7.11.3 Dependence on the symmetry index $n$ and the spatial dimension $d$. Universality classes ...293

7.11.4 Borderline dimensions .................. 294

7.11.5 Breakdown of the standard perturbation expansion

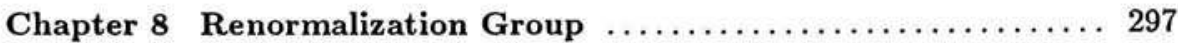

8.1 Modified perturbation scheme ............... 297

8.2 Perturbation expansion for the effective Hamiltonian .. 301

8.2.1 First order calculation $\ldots \ldots \ldots \ldots \ldots \ldots \ldots . \ldots 2$

8.2.2 Vertex functions $\ldots \ldots \ldots \ldots \ldots \ldots \ldots \ldots \ldots \ldots$

8.2.3 Second order calculation $\ldots \ldots \ldots \ldots \ldots \ldots \ldots, 305$

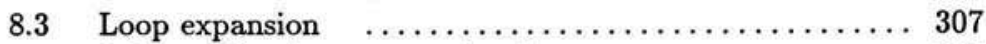

8.3.1 One-loop approximation $\ldots \ldots \ldots \ldots \ldots \ldots \ldots . \ldots 307$

8.3.2 Two-loop approximation $\ldots \ldots \ldots \ldots \ldots \ldots .309$

8.3.3 Large- $b$ limit and one-component field ....... 311

8.3.4 Remarks on the renormalization .......... 312

8.4 Large- $n$ limit and Hartree approximation $\ldots \ldots \ldots \ldots 313$

8.4.1 Hartree diagrams $\ldots \ldots \ldots \ldots \ldots \ldots \ldots \ldots . \ldots \ldots$

8.4.2 Large- $n$ critical behaviour ............. 314

8.4.3 Hartree approximation $\ldots \ldots \ldots \ldots \ldots \ldots \ldots . \ldots \ldots$

8.5 Renormalization-group ( $\mathrm{RG}$ ) recursion relations $\ldots \ldots \ldots 318$

8.6 $\mathrm{RG}$ in the one-loop approximation $\ldots \ldots \ldots \ldots \ldots \ldots . \ldots \ldots$

8.6.1 General features of the RG equations ...... 323

8.6.2 RG at the critical point $\ldots \ldots \ldots \ldots \ldots \ldots \ldots . \ldots \ldots$

8.6.3 Critical Hamiltonian and notion of fixed points (FPs) $\ldots \ldots \ldots \ldots \ldots \ldots \ldots \ldots \ldots \ldots \ldots \ldots \ldots \ldots \ldots \ldots$

8.7 Linearized RG in the one-loop approximation $\ldots \ldots \ldots 330$

8.7.1 Recursion relations and FPs $\ldots \ldots \ldots \ldots \ldots .330$

8.7.2 Linearization and exponents ............ 332

8.7.3 RG flow lines and classification of the scaling

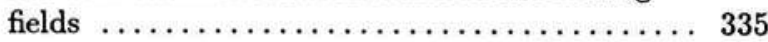

8.7.4 Remarks on the effect of the high-order interaction terms $\ldots \ldots \ldots \ldots \ldots \ldots \ldots \ldots \ldots \ldots \ldots \ldots$

8.7.5 Dimensional analysis $\ldots \ldots \ldots \ldots \ldots \ldots \ldots \ldots \ldots$

8.7.6 Differential RG relations $\ldots \ldots \ldots \ldots \ldots \ldots . \ldots \ldots$

8.8 RG and thermodynamics $\ldots \ldots \ldots \ldots \ldots \ldots \ldots \ldots \ldots \ldots$ 
8.8.1 Scaling form of the thermodynamic potential and classification of the scaling fields ....... 342

8.8.2 Scaling relations $\ldots \ldots \ldots \ldots \ldots \ldots \ldots \ldots \ldots \ldots$

8.8.3 Remarks on the crossover phenomena in the critical region $\ldots \ldots \ldots \ldots \ldots \ldots \ldots \ldots \ldots \ldots \ldots$

8.8.4 Scaling and $\epsilon$-expansion $\ldots \ldots \ldots \ldots \ldots \ldots \ldots \ldots$

8.9 RG in the two-loop approximation. ............. 351

8.9.1 Recursion relations to order $\epsilon^{2} \quad \ldots \ldots \ldots \ldots \ldots 352$

8.9.2 Fixed points $\ldots \ldots \ldots \ldots \ldots \ldots \ldots \ldots \ldots \ldots \ldots \ldots \ldots$

8.9.3 Exponents and stability properties. ....... 356

8.10 Other theoretical schemes and methods of calculation .. 359

8.10.1 Direct calculation of the critical exponents . . . 359

8.10.2 RG in three dimensions $\ldots \ldots \ldots \ldots \ldots \ldots \ldots 360$

$8.10 .31 / n$-expansion $\ldots \ldots \ldots \ldots \ldots \ldots \ldots \ldots \ldots \ldots \ldots \ldots \ldots$

8.10.4 $x$-space RG and references to other topics $\ldots .362$

8.11 Summary of RG and relations with other theories .... 362

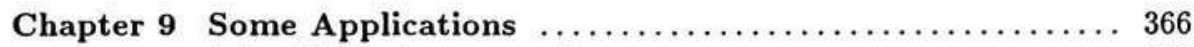

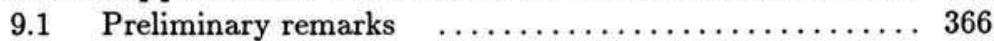

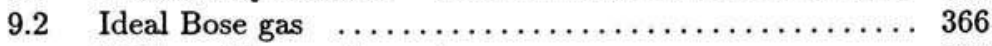

9.2.1 Thermodynamics $\ldots \ldots \ldots \ldots \ldots \ldots \ldots \ldots \ldots, 367$

9.2.2 Bose-Einstein condensation at finite

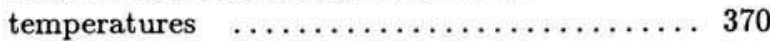

9.2.3 Zero-temperature condensation $\ldots \ldots \ldots \ldots \ldots .370$

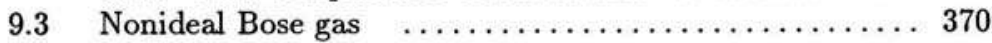

9.3.1 Notion of universality of the critical behaviour at finite critical temperatures ..... 370

9.3.2 Functional formulation $\ldots \ldots \ldots \ldots \ldots \ldots \ldots . \ldots \ldots$

9.3.3 RG results ...................... 374

9.3.4 Notes ......................... 378

9.4 Quantum critical phenomena and classical-to-quantum

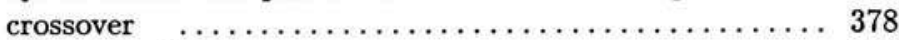

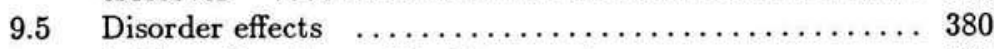

9.5.1 Random critical temperature $\ldots \ldots \ldots \ldots \ldots \ldots .381$

9.5.2 Extended impurities and long-range random correlations ...................... 383

9.5.3 Random fields ....................... 384

9.5.4 Technical remarks $\ldots \ldots \ldots \ldots \ldots \ldots \ldots \ldots . \ldots \ldots$

9.5.5 Ginzburg criterion $\ldots \ldots \ldots \ldots \ldots \ldots \ldots \ldots . \ldots \ldots$

9.6 Anisotropy, coupled order parameters and multicritical behaviour ..................... 387

9.6.1 Anisotropic systems . . . . . . . . . . . 387

$\begin{array}{llll}\text { 9.6.2 Mean-field analysis of anisotropic systems } & \ldots & 388\end{array}$

9.6.3 Cubic anisotropy in the mean-field approximation 
9.6.4 RG studies of anisotropic systems $\ldots \ldots \ldots \ldots 392$

9.6.5 Tricritical points and RG .............. 393

9.6.6 Bicritical and tetracritical points .......... 394

9.6.7 Lifshitz's and tricritical Lifshitz's points ..... 396

9.7 Critical properties of superconductors and liquid

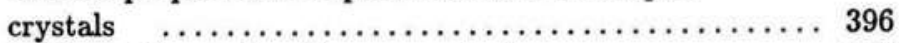

9.7.1 Conventional superconductors $\ldots \ldots \ldots \ldots \ldots . . \ldots 97$

9.7.2 Unconventional superconductors . . . . . . . 399

9.7.3 Layered superconductors $\ldots \ldots \ldots \ldots \ldots \ldots . . \ldots 99$

9.7.4 Fluctuations ...................... 400

9.7.5 Critical properties and the fluctuation induced weakly-first-order transition in superconductors $\ldots \ldots \ldots \ldots \ldots \ldots \ldots \ldots \ldots, 401$

9.7.6 Weakly-first-order smectic A-nematic phase transition ......................... 403

9.7.7 RG investigations ................. 404

Appendix: Useful Mathematical Formulae $\ldots \ldots \ldots \ldots \ldots \ldots \ldots . \ldots 4$

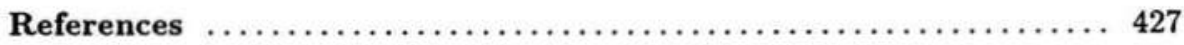

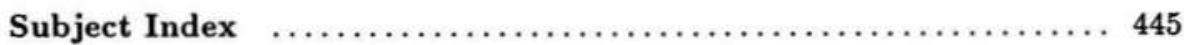




\section{Introduction to the Theory of Critical Phenomena}

